\title{
Signalling of rhizosphere micobiomes: Benign and Malign borders
}

\author{
Subhoshmita Mondal and Sibasish Baksi \\ Department of Chemical Engineering, Jadavpur University, Kolkata (India)
}

Keywords: Signalling, rhizosphere microbiome, plant hormones

\section{Introduction to Microbiome}

Signalling is a strongly influenced area trending to be applied in almost every focus of biological sciences. The part of signalling or communication from cellular level to a whole organism including plant as well as animal drags a vast diversity of wealthy structural compounds. There is immense demand for new bioactive compounds for the pharmaceutical, agro and food industries. Plant-associated microbes present an attractive and promising source. The concept of the microbiome and the significance it has to host health, diseases state, and the role of immune have been the hub of research that has led to advances in our understanding of the massive power of the small unseen majority of the microbes (Peterson Andrew H., 2013).

Before we say about microbiome - plant relation, it is important to first understand the working concept of the microbiome. Every organism on earth counts on their neighbours to sustain life. Microbiome can be considered a community of microorgasims who can prove to be loveable and hateful. The analysis of microbiome structure and function was protagonise in studies of human hosts and has been extensively documented as essential to genetic and functional capacity attributed to the host, comprehending aspects of metabolism and physiology. Plants are crowded with microbial organisms, counting those that colonize internal tissues, also those that adhere to external surfaces. The wide diversity of microorganisms in the soil rhizosphere is collectively plant-soil-associated microbes cover the plant microbiome. The intricate involvement of microbiome serves to plant health and as a tank of additional genes that plants can access when needed. 


\section{Who is there? What are they doing? — The Rhizosphere microbiome}

The core plant microbiome defines the habitat, which is extended over a series from the whole plant as an individual to particular regions (roots, leaves, shoots, flowers, and seeds), as well as interaction between roots and the adhering or surrounding soil-the rhizosphere. The rhizosphere shows the sector of narrow zones of influence created by the roots through their exudates and by the exudates of the microbiome within the soil matrix. The importance of epiphytic and endophytic lifestyles to the plant microbiome is critical.

Plants are colonized by an amazing number of microorganisms multiplies to cell densities much greater than the number of plant cells. Many studies have exposed plant-associated microorganisms can have reflective effects on seed germination, seedling vigour, plant growth $\&$ development, nutrition, diseases and productivity.

Conceptually rhizosphere commonly portrays the volume of soil influenced by roots also the root-soil interfaces. Rhizospheres are complex and highly-dynamic environments where the biogeochemical cycling of elements are steered by an enormous number of interactions between roots, minerals, organic compounds, solutes, gases and microorganisms drive. Immobile cations, such as phosphorus, potassium, and ammonium get depleted quickly in the rhizosphere, when more mobile ions can be restored (Peterson Andrew H., 2013)

The rhizosphere is a highly complex ecosystem consisting of the narrow zone of nutrient-rich soil that surrounds, and is influenced by, plant roots. It is heavily occupied by diverse microorganisms including fungi, bacteria, protists, nematodes, and invertebrates. A mixture of primary metabolites (organic acids, carbohydrates, and amino acids) and secondary metabolites (alkaloids, terpenoids, and phenolics) which are believed to shape, signal, interfere with, or in some way affect the rhizosphere microflora are secreted by plant roots. This release or exudation of lesion in the rhizosphere of a large amount of chemicals comes at an assortment 
of carbon and nitrogen for the plant, with eventual captivating and promoting beneficial microorganisms followed by hostile pathogenic or otherwise harmful ones. In particular rhizosphere microbiota molds the plants to adapt to the environment, and the establishment of a specific microbiota member in the rhizosphere can be said as niche colonization. The main attention of the rhizosphere microbiome or rhizomicrobiome is believed to be based on the chemical exudates, which also arbitrate interactions via signalling molecules produced and secreted by both plants and microbes. The extent of signalling between root and microbial exudates and its effect on rhizosphere microbial structure and function is a subject of ongoing research and unknown facts; specially, how the plant selects the rhizomicrobiome and most vitally the favourable microbial partners (Berendsen et al. 2012)

Scientists regard this aspect as underground interactions, signalling or communication highways, rhizosphere chemical language, and complex plant-microbe interactions, but two main questions still ponder - what comprises signalling in the rhizosphere and what are the responsible mechanisms? The shaping and recruitment of the rhizomicrobiome can be regarded as the occurrence of stimulation by the rhizodeposits or root exudates (approx. $10 \%$ of photosynthetically fixed carbon and $15 \%$ of total plant nitrogen) of microbial multiplication in the environs of roots. This is an active and key route for recruiting, shaping, and tuning microbial rhizosphere communities from the handful of microorganisms present in the soil, involving processes that support, restrict, or terminate microbial growth and activity (Mendes et al. 2013).

The group of bacteria have been always focused, but in the recent years wide survey of fungi and protists as well have helped in developing a much broader view of the rhizosphere microbiome. 
Starkey (1938) coined "rhizosphere effect", defined by collective processes taking place at the root-soil interface of a plant and comprise root exudation, microbial activity, genetic exchange, nutrient transformation and gradient diffusion. In living plants, organic carbon released by plant roots is decomposed to $\mathrm{CO}_{2}$ in a mechanism known as rhizosphere priming effect (Halder and Sengupta, 2015). The soil compartments adhered directly around the plant root contains a population of microorganisms. The large amounts of organic carbon released by the plant roots amplify microbial numbers and activities in the rhizosphere. A broad review explained, the major routes of rhizode position are loss of root cap and border cells, insoluble mucilage, soluble root exudates, volatile organic carbon, and flow of carbon to root associated symbionts, and death and lysis of root cells were. Soil microorganisms are chemotactically dragged towards the plant root exudates, after which they propagate in carbon rich environment (Lugtenberg and Kamilova, 2009; Jones et al. 2009; Mendes et al.2013).

As estimated, the plant carbon economy is still controversial with respect to approximately one-third to half of total assimilated carbon is allocated to below-ground, of which $15-25 \%$ is exuded from the roots into the soil to induce fast carbon-turnover in the rhizosphere (Halder and Sengupta, 2015). Due to rigorous carbon uptake by the roots, other nutrients in the rhizosphere are strongly limited. But in a root-free soil, all the nutrients except carbon are unlimited. The remains of easily available carbon and strong nutrient limitation, together, make the environment for rhizosphere strongly different from that of the root-free zones. The augmented microbial number and their activities in the rhizosphere compared to those in bulk soil are mainly due to the release of organic carbon by the plant roots.

Rhizodeposit are collectively known as the substances released by plant roots as and these compounds in general include the followings,

(i) Water-soluble ions and low-molecular-mass compounds such as monosaccharides, amino acids and organic acids lost passively along a concentration gradient. 
(ii) High-molecular-mass compounds such as carbohydrates, proteins and lipids dynamically transported along an electrochemical gradient.

(iii) Insoluble mucilage composed of polysaccharides and polygalacturonic acid.

(iv) A series of secondary metabolites such as antimicrobial compounds, nematicides and flavonoids and,

(v) Sugars and amino acids are thought to be released in the greatest quantities from leftovers of the dead and lysed root-cap and border cells.

As mentioned plant root exudates differ between plant species, with differences in rhizosphere microbiomes of different plant species are expected. It is studied from comparing plant-specific microbial communities isolated from roots, for example, wheat, ryegrass, bent grass, and clover. The roots of wheat, maize, rape, and barrel clover were shown to carry different bacterial communities. The growth stage of the plant affected bacterial community structures in rhizospheres of field grown potato. Also within a bacterial group like fluorescent Pseudomonas spp., plant species-specific rhizosphere populations could be isolated. More recent studies showed rhizosphere microbiomes were characterized based on direct extraction of total community DNA, which presented strong evidence for plant species-specific microbiomes (Inceoglu et al. 2013; Bakker et al.2013). 


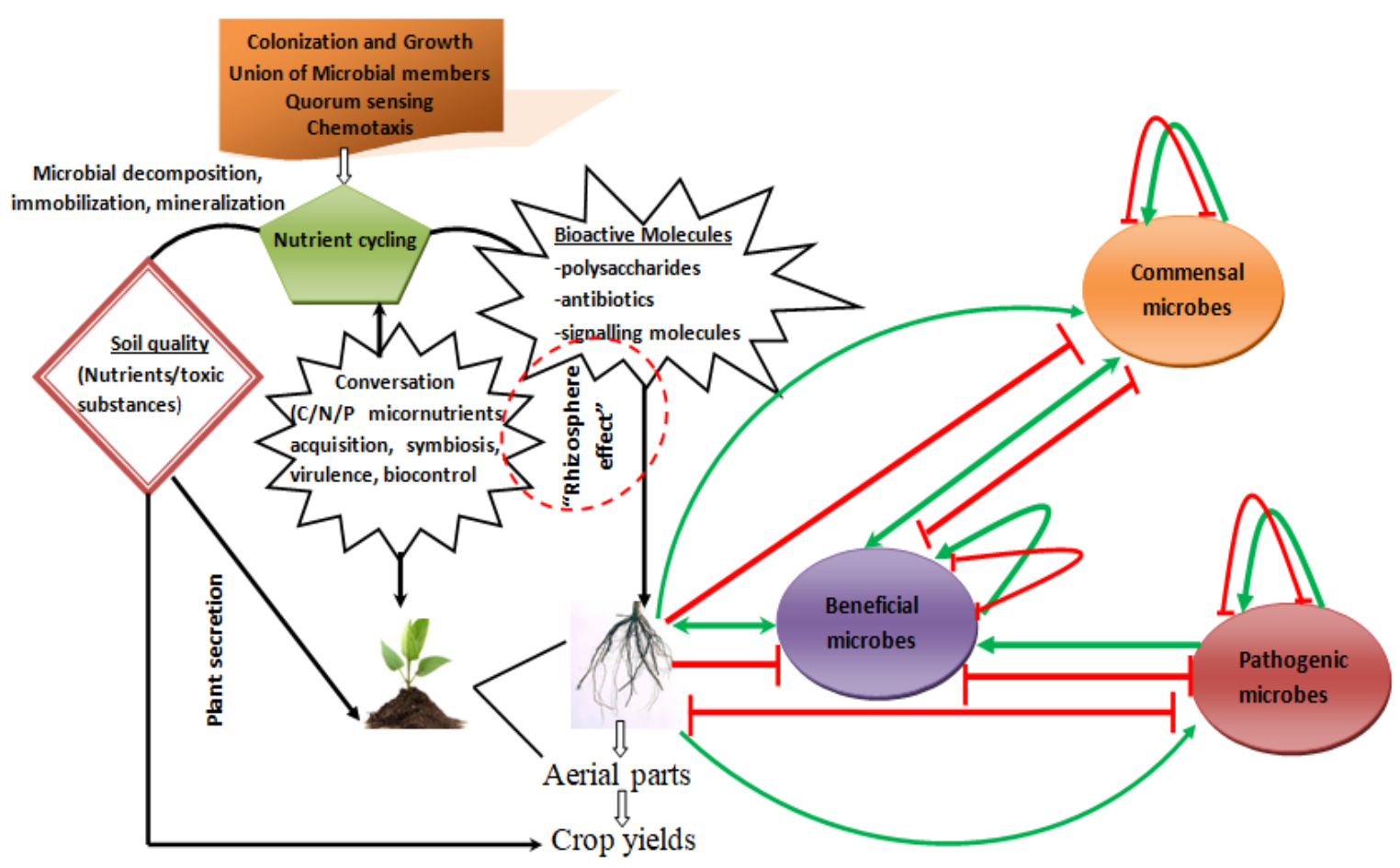

Figure 1. Flowchart of a combined rhizobiome communication where the influence of plants for activation of microbes via exudation of comounds stimulate (green arrows) or inhibit (red blocked arrows).

\section{Plant Metabolism}

-Metabolites (alkaloids, terpenoids, phenolics), amino acids -Plant growth promoting rhizobacteria -Volatile organic compounds - Lipo-chitooligosaccharides

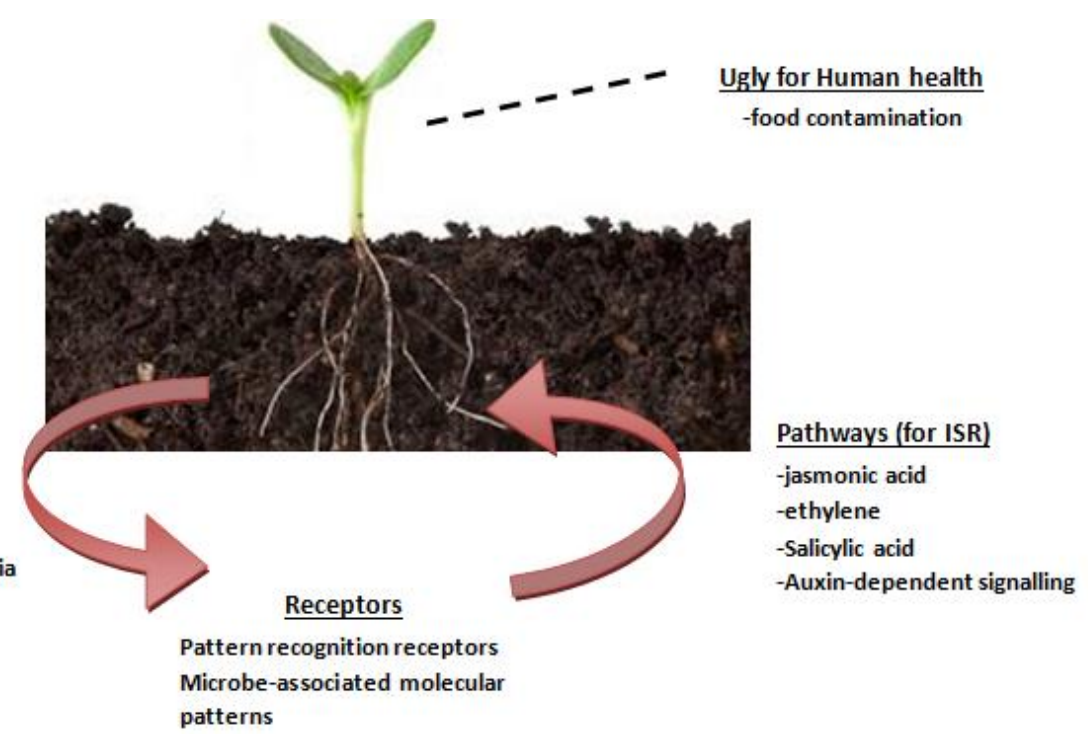

Figure 2: Essential carriers and routes for signalling effect of rhizomicrbiome. 
The current advances in molecular biology promoted the researches on nutrient acquisition of rhizobiome. An approach for mutagenesis of a blend of rice breeds showed heritability for micronutrient uptake, such as manganese and zinc, along with uptake of hazardous trace metals such as cadmium (Tanaka et al. 2016, Mommer et al. 2016). While much of the accumulated knowledge on plant nutrition and on the fate of nutrients in the rhizosphere in hydroponics experiments with a single crop have endorsed biodiversity-based agriculture sustainable intensification of agro ecosystem, and embrace the complexity of rhizosphere processes occurring in systems like intercropping. Positive plant-plant interactions with enhanced rootroot facilitation play a significant role for positive acquisition (Li et al. 2016; Zhang et al. 2016; Mommer et al.2016). They further demonstrated the greater performance of such intercropping systems than single crop systems under field conditions along a fertility gradient. They showed that the over yielding of the intercrops occurred at all levels of phosphorus fertility, reducing the stress gradient with more positive plant-plant interactions under low phosphorus conditions. However, when considering the properties of the rhizosphere, phosphorus fertility is a major cause for rhizosphere microbial communities. It was also observed that intercropping had a significant effect on microbial properties of the rhizosphere only at the higher end of the fertility gradient. Hence, soil fertility has a significant impact on the outcome of root-root and root-soil interactions, which requires to be further investigated in various types of ecosystems. Soil fertility is linked to carbon sequestration via soil organic matter (most dynamic portion) which can be dissolved and circulated within soil. Changes in nutrient availability and uptake may affect dissolved organic matter, changes in decomposition rate (Robroek et al. 2016; Mommer et al. 2016) the detected effect of the functional types of vascular plants on microbial enzymatic activity and the composition of dissolved organic matter.

Rhizosphere is more than exchanges between roots and nutrients, since water is also an essential driver. This focuses on two features, the impact of the root age and function on root 
water uptake and rhizosphere biophysics. In spite of surprising scarcity of data on root age distribution with depth, and on the fate of roots and root functioning, the current theoretical developments with experimental quantification like hydraulic conductance variation have supported the predicted evidences backing up the models. The functional properties possibly influencing water relationships in the rhizosphere affected by root age are however many: from axial hydraulic conductance, occurrence of apoplastic barriers, secondary growth or distribution of aquaporin expression to decay of root cortical cells and mucilage production (Vetterlein and Doussan 2016, Mommer et al. 2016). Meanwhile, researchers overcame this gap by measuring the water repellence of root-exuded mucilage (plant specific polysaccharides) on maize plants under different soil conditions. This combination of measurements and modelling showed, beyond a threshold concentration that decreased with increasing particle size and decreasing matric potential, root mucilage can turn the rhizosphere hydrophobic, finally resulting in a spectacular reduction of water flow at the root-soil interface. This work has showed a direction to the field of rhizosphere biophysics and its major impact on the fate of water, nutrients and other solutes in the root environment, at various levels from confined state to the whole root system scale (Ahmed et al. 2016).

\section{Signal conversation in the rhizosphere microbiome}

The upcoming study to know the communication among plants, among microbes, from plants to microbes, and from microbes to plants, describes the signalling in the rhizosphere. It particularly focuses on the modulation of plant metabolism by microbes, with a specific highlight on the impact of bacterial inoculation on plant volatiles that leads plant stress alleviation. We know an emerging mechanism of communication between bacteria at both intra species and interspecies levels - as quorum sensing. It allows bacterial populations to synchronize their behaviour to cell density by exchanging numerous signal molecules. Quorum-sensing is involved in several bacterial regulations, such as biofilm formation, 
virulence in pathogenic bacteria, and production of antimicrobial compounds by biocontrol plant growth promoting rhizobacteria (PGPR). There is also existence of direct bacterial quorum-sensing signals on plants which can trigger induced systemic resistance and improve plant defence against pathogens (Venturi and Keel 2016). Some antibiotics when present in low concentration can also be involved in communication between microbial populations, triggering gene expression regulation in the surrounding bacterial cells. The gene expression is affected even across inter kingdom domains; signals emitted by microbes have thus vital roles in shaping rhizosphere microbial communities and controlling their functioning. Interactions between microbes are regulated basically by root exudates, having a high diversity of primary and secondary metabolites (include sugars, organic acids, amino acids, phenolic or terpenoid compounds) which can affect the rhizosphere bacterial populations by acting as nutrient or signal molecules (van Dam and Bouwmeester 2016). Through their nutritive effects, exudates will affect the structure of soil microbial communities, mediating cell division of bacteria available to catabolize them. With the progress in metabolic networks identification, major advances were recently made based on the characterization of plant root exudate composition and their differences among plant genotypes, environmental conditions or even experimental conditions during harvest. The microbe-plant chemical dialogues are very well characterized in symbiotic interactions between legumes and nitrogen-fixing rhizobia or in plant mycorrhiza, than in plant-PGPR interactions. Plant-microbe signalling involves a wide range of molecules produced by the microbe partner, some being directly controlled by plant growth and development; these consist of phytohormones like auxin, cytokinin, and gibberellin. Studies focused on promotion of plant-PGPR cooperation by ethylene signalling, which is a plant volatile hormone produced in greater amounts when plants face adverse conditions, and may inhibit the growth of plant roots. The hormone is synthesized from 1-aminocyclopropane-1carboxylate (ACC) with the help of ACC oxidase. Next ACC deaminase harboured by bacteria, 
hydrolyze ACC, decreasing plant ethylene content and thus enhancing root growth. In addition, AcdS is the gene encoding for ACC deaminase synthesis that can protect the plant against certain damage. The endophytes isolated from fruits and vegetables (including carrots) produce ACC deaminase modifies stress-induced volatile metabolic profiles of carrots (flavour of carrots). As the expression of the ACC deaminase synthesis-encoding AcdS gene is activated in presence of ACC, this bacterial enzymatic activity introduce stimulation of plant growth that depends on the levels of exuded ACC in the rhizosphere. On the other side, maize genotypes differ in their ability to promote the expression of the AcdS gene of an inoculated PGPR, also the ethylene pathway is strongly activated by stress conditions.

The other ways affecting the rhizomicrobiome occurs through the detection and response to low molecular weight compounds of either plants or microbes, ensuing in a cellular responses which is not only restricted to the catabolism, transformation, or resistance of the compounds being sensed. This demands a regulatory response/cascade which eventually leads to the transcription of loci in response to a particular compound. This is what believed to satify the definition of signalling yet, to further precise our understanding of this rapidly growing research field, signalling mechanisms represent three categories known to occur in the rhizosphere:

(a) Microbial intraspecies and interspecies signalling, occurence mainly via quorum-sensing (QS) signal molecules allowing microbial populations to form and synchronize their behaviour.

(b) Signalling from plants to microorganisms via small plant-secreted molecules, which have been occupied in several specialized symbiotic relationships.

(c) Signalling from microorganisms to plants documented so far by microbial secreted compounds affecting plant gene expression, root structures, and plant defense responses.

\section{Routes of Communication- Rhizobiome}




\section{Microbe-Microbe Communication}

Many microorganisms synthesize signalling compounds to synchronize their gene expression triggered from cell density or population which defines quorum sensing. Foremost, many groundbreaking studies on QS have been established backing up with models of plantmicrobe linkages regulating diverse processes such as the production of virulence factors, synthesis of secondary metabolites, formation of biofilms, conjugation, and motility. The different types of signals in a multiple QS systems are generated by microbes belong to a wide range of chemical classes. It is not the scope here to review exhaustively the QS signals being produced by the rhizomicrobiome but instead to inform the reader of the major trends and directions within the topic of rhizosphere signalling. The QS signals caused by the rhizomicrobiome are likely to play an essential role in shaping and stabilizing the rhizosphere microbial community as well as affecting plant development. The rhizosphere microorganisms are likely to involve cell-cell signalling among many strains isolated from the rhizosphere reported to produce QS signals. It has become clear that a diversity of proteobacterial rhizosphere isolates act in response to $\mathbf{N}$-acyl homoserine lactone (AHL) QS signals, including strains fit into species or genera of Pseudomonas chlororaphis, Pseudomonas putida, Pseudomonas syringae, Burkholderia, Serratia, Erwinia, and Ralstonia, as well as rhizobial species. At inter kingdom levels also AHLs evolved activate signals influencing plant gene expression, the impulsion of systemic plant resistance, and affecting plant growth and development. The different types of molecules, pyrones and dialkylresorcinols produced by Gram-negative bacteria have been exposed which are recognized by LuxR proteins very closely related to the AHL-responsive LuxR family; but it is unknown whether these are produced by rhizobacteria. Another class of QS signals in Gram-negative bacteria is the diffusible signal factor family (cis-2-unsaturated fatty acids); also discovered including rhizosphere-residing species such as Burkholderia spp. and Stenotrophomonas maltophilia. 
Interestingly, bacterial signals from diffusible signal factor have been recently determined to bring out innate immunity in plants, hence acting as inter kingdom signals. Meanwhile, Grampositive inhabitants in the rhizosphere produce pheromones (peptides) as QS signalling molecules; which control many regulatory roles both at the intra- and interspecies level. An attractive fact proposed that antibiotics are potential class of bacterial signals to function, at low and non-inhibitory concentrations, as intra- and interspecies signalling molecules. An important characteristic determined is the ability of microorganisms to release a series of volatile organic compounds (VOCs), among them many are still unrelated. However, the chemical structures of several VOCs have been reported and they are typically small molecules (100-500 Da, these are generally alkenes, alcohols, benzenoids, aldehydes, ketones, terpenes) derived from diverse bacterial and fungal species. Microbial VOCs are supposed to play an important role in long throw of distant interactions in microbial communities which involve in microbe-microbe as well as plant-microbe interactions in the rhizosphere. When implicated as microbe-microbe interactions, they act as chemical weapons by disporting antimicrobial activity; alternatively they interfere and shape up other QS systems. VOCs can act in intraspecies as well as interspecies signalling by conducing gene expression and subsequently influencing microbial biofilm formation, virulence, and stress tolerance, in addition regulating plant growth and stress resistance, plant immunity, and expression of plant genes involved in defense and hormonal signalling pathways. However, researches on VOCs implicated in several signalling mechanisms with a probable impact on the rhizomicrobiome are at its early phase. It must be noted as well that many rhizosphere microorganisms have been found to produce and respond to QS signals, and it is likely that even if not all are capable but at least respond to one class of microbial signals. This intense and intricate communication among microorganisms recruits in shaping the microbial community in the rhizosphere. These 
microbial signals could hence be an important means to use in the future for couturing the rhizomicrobiome (Venturi and Keel 2016).

\section{Plants -Microorganisms Communication}

Plants serve as hosts to the rhizosphere signifying an overwhelming diversity of communal mutualist, or parasitic microorganisms, where secretion of phytochemicals is vital for these plant-microbe interactions. The endosymbiotically growing nitrogen-fixing rhizobial bacteria forming legumes is the best depicted signalling network between plant and rhizosphere microorganisms with different. Upon signal exchange, rhizobial bacteria colonize root nodules, fix atmospheric nitrogen inside, and transport usable forms of nitrogen to plants, thereby aiding their growth. The flavonoid compounds (2-phenyl-1,4-benzopyrone derivatives) are the first signal molecules discovered to induce bacterial nod genes during the exchange between the host plant and its rhizobial symbiont. These bacterial nod genes are liable for producing and secreting lipo-chitooligosaccharides (LCOs), or nodulation (Nod) factors, which are the foremost signal molecules for initiating nodule formation. The mechanism pathways of LCOs are then professed by the plant via receptor kinases at the root epidermis, thereby activating a well-characterized signal cascade leading to nodule formation. In addition, LCO signals are involved in symbiosis with plants and arbuscular mycorrhizal fungi (AMF or AM), one of the most important rhizosphere mutualisms. This assistance is therefore well established and capacitates nutrient exchange between the two partners. The fungus increases the ability of roots to access nutrients from the soil, particularly immobile phosphates; in return the plant provides carbohydrates to the fungus. It was recognized that a set of legume mutants of LCOinduced signalling are incapable to undergo rhizobial inter kingdom communication which are also hindered in mycorrhizal symbiosis. It has been strongly commended that mycorrhizal fungi produce similar LCO-type signalling molecules Myc-LCOs, and it is most expected during evolution rhizobia co-opted the mycorrhizal signalling machinery. In spite of the 
ecological importance, mechanisms of the signalling underlying this symbiosis at molecular level are only partially understood. The chemical dialogue involved in the communication is assumed to be continuous with established long-lasting colonization, but it seems that it is not very specific because there is no plant host specificity. This signifies that either the plant signals are confined throughout the plant kingdom or perhaps that a range of plant compounds are involved.

Substantially, some molecules secreted from plant roots act as signals for AM fungi; as such, the strigolactone plant hormones are measured to be one of the primary signals for initiating AMF symbiosis. Strigolactones have been associated with multiple functions because they can act as an ex planta stimulus for mycorrhizal hyphae and also act as plant hormones snooping with auxin transport. Intriguingly, root-parasitic plants such as Striga spp. and Orobranche spp. capture signals produced by strigolactone to sense their host and induce seed germination surrounding area of the roots. The scenario of association of several molecules which mediates multiple functions also provokes signals in the rhizosphere and has a role in planta. It have been recently drawn out that cutin monomers are specified as a class of plant signalling factors which play a crucial role for AMF stimulation. Two loci, RAM1 and RAM2 (required for arbuscular mycorrhization), have been recognized in Medicago truncatula mutants badly affected in AM symbiosis; they encode a GRAS domain transcription factor and a acyl transferase, respectively, responsible for the production of cutin monomers. Importantly, signals derived from cutin monomer also play a role in appressoria formation in aerial fungal pathogens. Strigolactones and cutin monomers are the cases in points that take part in plant signalling perceived by beneficial as well as pathogenic organisms. Rhizobia and AM fungi are examples of close symbiotic relationships between plants and microorganisms in the rhizosphere, and the signalling pathways have been meticulously studied to provide data on signals and cascade mechanisms. Because many different microorganisms live in the 
rhizosphere, even if not in such close association with plants, inquisitively query arise on the role of plant secreted signals which affect gene expression of members of the rhizomicrobiome. After strains of plant-pathogenic xanthomonads, rhizosphere rhizobia and pseudomonads were studied, demonstrating that this inter kingdom signalling system is involved in regulating traits important for in planta colonization, it was found in rhizosphere proteins called PsoR is involved in transcriptional regulation in response to plant compounds of various antimicrobial related genes and in biocontrol. In this common plant-bacterium signalling system, the key step is now to identify the plant signals which is exciting this communication and is expected to be prevalent low molecular weight plant secondary metabolites. These plant secondary metabolites comprises of terpenoids, phenylpropanoids, and fatty acid and amino acid derivatives and are assumed to cross membranes freely and get released into the atmosphere or soil. Their main functions are to defend plants against herbivores and pathogens or implement reproductive advantages. In the roots, volatiles can act as antimicrobial substances or as attractants for antagonist effect of root-feeding herbivores. A well-studied example of an attractant is the volatile (E)-b-caryophyllene emitted by the roots of maize responds when fed by the larvae of the Western corn rootworm (WCR). This volatile is highly striking to an entomopathogenic nematode which paralyses and kills WCR within a few days. This illustrates very well the signalling role of volatiles in roots and they can be also elucidated in many other tritrophic interactions. Like plant growth-promoting rhizobacteria (PGPR) of plant-beneficial rhizosphere microorganisms for the genera Pseudomonas, Bacillus, and Azospirillum, similarly plant growth-promoting fungi (PGPF) involves Trichoderma and non-pathogenic fusaria. At first Mycorrhiza, rhizobia, PGPR, and PGPF all are recognized as non-self by the plant, which uses precised pattern recognition receptors (PRRs) to spot preserved microbe-specific molecules, termed microbe-associated molecular patterns (MAMPs) consist of lipopolysaccharides, peptidoglycans, flagellin, and chitin. The MAMPs 
are useful in triggering a local basal immune defense in the roots, which then can interpret systemic defense responses that are governed by regulatory networks causing signals via the plant hormones salicylic acid, jasmonic acid, ethylene, and others. The plant defense response, usually induced by root-colonizing PGPR and PGPF, is known as induced systemic resistance (ISR) and acts methodically against various foliar pathogens and even leaf-feeding insects. The jasmonic acid and ethylene signalling pathways govern ISR, and is distinct from the systemic acquired resistance (SAR) which is generally induced by pathogens where salicylic acid signalling takes part. The innate immune response triggered by rhizosphere beneficial microorganisms is comparatively mild and is based on a process called priming which initializes the plant, upon sensitization by the microbes, to respond more efficiently to abiotic and biotic stress such as attack by leaf pathogens and pests. Rhizosphere microorganisms explain plant responses not only by means of MAMPs and effector proteins but also through diverse signalling molecules. The Nod and Myc factors remitted by rhizobia and mycorrhiza, are best examples of microbial inter kingdom signalling molecules, combinedly they suppress salicylic acid-dependent antagonist responses and initiate a symbiosis signalling pathway. In fact, ectomycorrhizal fungus Laccaria bicolour (releases the $7 \mathrm{kDa}$ protein MiSSP7 from its hyphae upon root contact) produce small secreted proteins (SSPs) that perform as mutualistic effectors endorse mycorrhization by changing hormonal signalling pathways in their plant host. The mini-protein then goes into the host cells, clusters at the nucleus, and interacts with plant hormone co-receptors to countercheck jasmonic acid signalling and promote symbiosis. An SSP mycorrhization inter colliding with hormonal signalling was also identified in the arbuscular mycorrhizal fungus Rhizophagus intraradices. However, it was stated that PGPR also release various potential signal molecules guiding inter kingdom signalling properties by influencing plant gene expression, defense responses, and developmental processes, but their exact mode of action and plant targets remain to be unclarified. Earlier published reports on 
extensive changes of protein outlines in roots of Medicago trunculata and on gene expression outlines in roots and shoots of Arabidopsis thaliana followed root conductance with structurally different AHLs. The production of AHL by rhizobacteria may encourage the plant host to spread out strategies to interfere with these bacterial signals, such as by producing AHL mimics, by blocking their uptake and transport or by destroying them. On a complete note, the less studied bacterial QS molecules with inter kingdom signalling properties exemplify Xanthomonas with diffusible signal factor (DSF) and cyclodipeptides, in other words diketopiperazines (DKPs), released by Pseudomonas aeruginosa, which sense salicylic acid, absicic acid, or auxin-reactive gene expression in planta. 2,4-Diacetylphloroglucinol (DAPG) existing at sub-inhibitory concentrations on roots or in rhizosphere soil, commenced salicylic acid and ethylene signalling-dependent induced systemic response against fungal and bacterial leaf pathogens in Arabidopsis. DAPG allowed to perceive on root development, and thus expressed that this occurs via an auxin-dependent signalling pathway. Similarly, phloroglucinols, Pyocyanin (a phenazine antibiotic) produced by $P$. aeruginosa, also induces ISR and modulates root development likewise. 2,3-Butanediol (2,3-BD) released by Bacillus PGPR strains was the first microbial VOC effecting plants, improve the growth of Arabidopsis and to induce ISR en route for phytopathogenic Erwinia via ethylene signalling pathways in the model plant. 2,3-BD produced by a Pseudomonas chlororaphis PGPR with induced ISR plays antagonistic effect on same pathogen in Nicotiana tabacum. Furthermore 2,3-BD produced by a root endophytic Enterobacter strain elevates the resistance of maize plants against a fungal leaf pathogen beside, affecting tritrophic interactions with a herbivorous insect and its parasitoid. Indole, one more bacterial signalling VOC produced by various PGPRs, affects root development in Arabidopsis via the auxin signalling pathway. Interestingly, indole released by plants also functions as a effective volatile signal initiating the producer and neighboring plants against invasions from herbivorous insects. A further class of microbial 
molecules with mutual signalling properties studied are phytohormone-like compounds, including auxins, gibberellins, and cytokinins, that are not only released by PGPR and PGPF but also by bacterial and fungal pathogens with impacts on growth, organ development, immune responses, and hormonal signalling in plants. This has been ascertained, ascaroside pheromones produced by plant-parasitic nematodes stimulated defensive gene expression and resistance to microbial infections in Arabidopsis and other host plants. Taken together, the effects take place locally in the root and systemically throughout the plant body, implying the involvement of root-shoot signalling events. A major confront will be to disclose the fact, whether the molecules observed and considered are indeed signals specific, which are recognized by dedicated plant receptors, or whether they function instead as priming agents that induce a general (mild) stress response, which can be interpreted by the plant host and distinguished from responses induced by MAMPS and effectors. It is known that the rhizosphere microbiome is extremely diverse and produces a huge diversity of compounds; a generic perception of these molecules as priming agents appears more plausible. (Quiza et al. 2015)

As shown in Figure 1, prior to infection, the deleterious microbes compete with many other microbes in the rhizosphere for nutrients and space. In this battle for resources, favourable microbes bound the success of the pathogen through production of biostatic compounds, consumption of micronutrients or by stimulating the immune system of the plant. Most microbes might neither affect the plant nor the pathogen directly because they occupy ecological niches (symbiotic microbes), but involve every other organism to a certain extent through a complex network of interactions. Root exudates and other rhizodeposits stimulate and/or inhibit various microbes. Nonetheless, all active microbes affect other microbes and through a complex of interactions indirectly affect either plant or pathogen the plant through infection or production of phytotoxic compounds. 


\section{The benefice and bull effects - Plant and Human health}

Consequently, so far it is imposed that rhizobiome directly and indirectly influence the composition and productivity (i.e. biomass) of assemblage of natural plants. Hence, richness of microbial species below the ground has been portended for above the ground plant diversity and productivity. Additionally the range below the ground may act as depositors for maintaining plant productivity under different environmental conditions. Due to their sensation to small changes in abiotic conditions, including environmental stress and concernment, soil and rhizosphere microbes are considered as bio-indicators of soil quality. It is also important to describe facts which show impact of rhizosphere microbes on plant growth and human health. The following sections say about the good and ugly faces of rhizomicrbiome.

\section{Conducive behaviour}

The adjuvant sides of rhizobiome support plant growth, protect plants from pathogen attack by a range of mechanisms, involve biofertilization, stimulate root growth, rhizoremediation, and control abiotic stress and disease. These mechanisms are well documented for the genera belonging to the Proteobacteria, Firmicutes and Planctomycetes..

The representatives of the rhizosphere microbiome can significantly influence the nutrient acquisition by plants. Well known examples are the nitrogen-fixers rhizobia and the mycorrhizal fungi that facilitate phosphorus uptake which also takes part as symbionts for translocation of nutrients and minerals from soil to the plant, for soil physical development and generating stable soil aggregates, and for suppression of soil-borne plant pathogens. On the basis of greenhouse experiments and 16S rRNA sequencing reports, Bradyrhizobium, Rhizobium, Burkholderia and Achromobacter species were competent to nodulate cowpea and efficient in biological nitrogen fixation. Extensive researchers have found that rhizosphere microorganisms can also facilitate the uptake of specific trace elements such as iron, which is plentiful in soil but when subjected under unbiased to alkaline conditions, it exists mostly in 
the insoluble ferric oxide state which is not accessible for microbial growth. Due to the scarceness of available iron in many microbial habitats as well as the toxicity of free iron at higher concentrations, employ a variety of mechanisms to regulate at intracellular level by secretion of siderophores. From the host side either of two strategies is followed, plants act in response to iron limitation by increasing the solubility of inorganic iron in the rhizosphere or by releasing phytosiderophores that are afterwards transported back into the root tissue by a specific uptake system. In addition to this various studies have anticipated a supplementary strategy of iron acquirement by plants for using chelated iron to microbial siderophores. This effect was exemplified in experiments with fluorescent Pseudomonads which promoted iron nutrition by means of siderophores not only for Graminaceous plants but also for dicotyledonous plant species. Besides rhizoferrin, a fungal siderophore produced by Rhizopus arrhizus, was brought to be an efficient transporter of iron to plants with competence that was comparable to that of synthetic chelates. Bacillus subtilis GB03, rhizobacteria are also able to turn on the plant's own iron acquisition machinery. In Arabidopsis GB03 up-regulated transcription of the Fe-deficiency-induced transcription factor 1 (FIT1), thus inducing the ferric reductase FRO2 and the iron transporter IRT1. Members of bacterial communities play crucial role in releasing the nutritive cations from soil minerals necessary not only for their own nutrition but also for plant nutrition. Bacteria acclimatizing mineral have been isolated under various environments, and particularly from environments with abundant rhizosphere and ectomycorrhizosphere which can supply to plant growth in nutrient-poor soils (Turner et al. 2013).

By supporting plant growth under biotic stress, the rhizosphere contributes the front-line of protection for plant roots against attack by soil borne pathogens. The community members of rhizosphere microbiome can obstruct soil-borne pathogens before and during primary infection, and secondly when it multiplies on and in root tissue. The main mechanisms by 
which rhizosphere microorganisms withhold plant pathogens are antibiosis, competition for trace elements, nutrients and microsites, parasitism, intrusion with quorum sensing affecting virulence, and induced systemic resistance. The rhizomicrobiome comprising of most among rhizobacteria and rhizosphere fungi, both groups are well known as prolific producers of antimicrobial metabolites that inhibit the growth or activity of competing microorganisms. Most fungal and bacterial biocontrol strains like Agrobacterium radiobacter produce more than one antibiotic compound with overlapping or different degrees of antimicrobial activity. Agrobacterium radiobacter release bacteriocins such as agrocin 84 exhibiting antibiotic activities against closely related genera. Interestingly, an observation which moved to a new direction in research on the natural functions of antibiotics as well as antibiotics compounds function in a concentration-dependent manner, act as growth inhibitors at high concentrations and as mediators of intercellular signalling at low concentrations. Although VOCs (are small molecules < 300 Da with high vapour pressures able to diffuse through the water- and gas filled pores in soil) appear to represent a small proportion of the total number of metabolites produced by fungi and bacteria, their unique properties have been proposed to play essential functions in long-distance communication in the rhizosphere and in soil ecosystems with modulation in plant growth. Diverse bacterial community including species like Stenotrophomonas maltophilia, Serratia plymuthica, Pseudomonas trivialis, Pseudomonas fluorescens, Bacillus subtilis and Burkholderia cepacia produce VOCs that restrain mycelial growth of fungal plant pathogens. Most work on VOCs till date conducted with effects of specific abiotic conditions, where difference in the number and spectrum of volatiles produced by a Xanthomonas species were grown in broth culture and on solid agar media. Also it has been set up that the volatiles produced in soil enhanced with artificial root exudates without amino acids had strong antibacterial effects but meek antifungal effects, whereas volatiles produced from root exudates complemented with amino acids had strong antifungal effects. Further, it 
was observed, bacterial volatiles may support growth of ectomycorrhizal fungi, regulate mycorrhizal network establishment and the tri-partite interactions between bacteria, fungi and nematodes. With reference to this context, Paenibacillus polymyxa and $\boldsymbol{P}$. lentimorbus indicated strong antifungal activities, thereby interfering with the interactions between Meloidogyne incognita and Fusarium oxysporum with associated nematode invasion of tomato plants. Moreover, dimethylsulfide interfered with quorum sensing of phylogenetically different bacteria; suppress the transcription of the $\mathrm{N}$-acylhomoserine lactone synthase genes. The phytohormones like jasmonic acid (JA) and ethylene (ET) regulate systemic resistance response induced in plants by beneficial rhizobacteria, while some bacterial strains such as, and Arabidopsis induce systemic resistance via the salicylic acid (SA)-pathway instead of JA/ET pathway. Furthermore, quorum-sensing molecules from rhizobacteria can provoke a range of plant responses, as well as responsible for the activation of various defense-related genes such as MPK3, MPK6, WRKY22, WRKY29 and Pdf1.2. By combining metabolic pathways and transcript profiles, two distinct strains of $P$. fluorescens minimised the host plant's carbon gain, but provided a beneficial fitness when the plants were subjected with the pathogen $P$. syringae. These studies indicated that rhizobacteria can have varied and reflective effects on the immune response and physiology/metabolism of the host plant, thus enhancing the production of known secondary metabolites but also inducing the biosynthesis of structurally unfamiliar metabolites. The activities of mystic plant compounds induced by rhizobacteria should be pursued and identified to resolve their hypothetical functions in induced systemic resistance and other physiological processes.

It has been postulated that the rhizosphere microbiome contributes to the plant growth of some plant species to survive under extreme conditions to overcome abiotic stress. Therefore, it is followed by an example of Achromobacter piehaudii ARV8, a soil isolate obtained from an arid and saline environment appreciably increased the biomass of tomato and pepper seedlings 
exposed to fleeting drought stress. The rhizobacteria equally supported plant growth under flooding conditions. It is well known; halotolerant bacteria succeed to survive under salt stress conditions and in association with the host-plant are able to express behaviours that promote plant growth, example, the rhizosphere of wheat plants grown in a saline zone, out of 130 rhizobacterial isolates, 24 were tolerant to relatively high levels $(8 \%)$ of $\mathrm{NaCl}$. All among the 24 salt tolerant isolates produced indole-3-acetic acid, 10 of the isolates solubilised phosphorus, 8 of them produced siderophores, 6 released gibberellin and 2 isolates contained the nif $\mathrm{H}$ gene, collectively indicated their potential for nitrogen fixation. More than a few obtained isolates improved plant growth under saline stress and reduced production of ethylene by means of ACC deaminase activity (Siddikee et al. 2010). Newly isolated halotolerant diazotrophic bacteria from roots, harbouring indole acetic acid (IAA) production, phosphate solubilization, and 1-aminocyclopropane-1 carboxylic acid (ACC) deaminase activity were identified as Salicornia brachiate, Brachybacterium saurashtrense sp. nov., Zhihengliuella sp., Brevibacterium casei, Haererehalobacter sp., Halomonas sp., Vibrio sp., Cronobacter sakazakii, Pseudomonas spp., Rhizobium radiobacter, and Mesorhizobium sp. (Jha et al., 2012). Accordingly, several mechanisms illustrated rhizomicrobes may change plant physiological reaction under saline stress results into water homeostasis by osmolyte assortment, plant energetics by modifying the source-sink relations, roots uptake of toxic ions and nutrients by changing host physiology, modifying physical blockages around the roots, or by straight way reducing foliar accumulation of toxic ions, and salt tolerant crop by deflecting hormonal root-shoot signalling. It is interesting to note that regardless of the impact of low temperatures on nodule formation and nitrogen fixation, inhabitant legumes in the high arctic can nodulate and fix nitrogen at rates comparable to those related for legumes in temperate climates. Another area of interest is that in agriculture and horticulture, microbial inoculants enhance growth of plants under cold conditions, for example, Burkholderia phytofirmans 
PsJN improved grapevine root growth and physiological activity at temperatures down to $4^{\circ} \mathrm{C}$. During co-inoculation with Bradyrhizobium japonicum, Serratia proteamaculans stimulated soybean growth at $15^{\circ} \mathrm{C}$, the temperature at which soybean nodule infection and nitrogen fixation is usually inhibited. To spot mechanisms involved in plant growth promotion at low temperatures, cold tolerant mutants of strains like, Pseudomonas fluorescens $\left(\right.$ at $\left.10^{\circ} \mathrm{C}\right)$, Pseudomonas corrugate (at $4^{\circ} \mathrm{C}$ to $28^{\circ} \mathrm{C}$ ) were selected for their ability to solubilise phosphorus and to promote plant growth. But both studies, did not mention any identified genes associated with cold tolerance and phosphorus solubilization. Other abiotic factors that may unfavourably affect plant growth are $\mathrm{pH}$ and high concentrations of toxic compounds. In cases of $\mathrm{pH}$ stress challenges, it was demonstrated that foliar lesions incited on corn growing in a low-pH soil drastically reduced on plants subjected under 2,4-diacetylphloroglucinol (DAPG)producing Pseudomonas fluorescens strain. This was the first fact that introduced DAPG producers as in addition to pathogen controllers, can also act to restructure abiotic stress factors (Berendsen et al. 2012, Mommer et al. 2016).

The flourishing area of efficient bioremediation methods as an alternative for excavation and incineration promoted the presence of pollutants in soil. The idea of rhizoremediation is a combination of phytoremediation and bioaugmentation. It is a promising strategy to clean polluted sites, where exudates of plants stimulate the survival and finally action of rhizobacteria that degrade pollutants. An evidential study, using a split-root model and a combination of biochemical methodologies, showed that Trifolium and other legumes act in response to polycyclic aromatic hydrocarbons contamination in a systemic mode. Verrucomicrobia and Actinobacteria were richer in the contaminated rhizospheres and the $\beta$-Proteobacteria Denitratisoma was considerably increased in the presence of the contaminant, signifying that this genus may be vital in the rhizoremediation course. Beside fungi are also considered key players in rhizoremediation of hydrocarbons as shown by inoculation of the endophytic fungus 
Lewia sp. in the rhizosphere of Festuca arundinacea (Berendsen et al. 2012, Mendes et al. 2016).

To conclude all the communal members of the rhizosphere microbiome, they have proved to ease biotic and abiotic stresses on plants, presenting an environmentally sound alternative for genetic engineering and plant breeding. However, successful performance of microbial inoculants is still in its early years due to multiple limitations with uneven efficacy across environments and different plants species, partial shelf-life. To resolve this tenacity, more basic knowledge is necessary on how beneficially rhizosphere microbes communicate with the host plant, involving molecular and metabolic changes induced in plants, also how microorganisms affect the population dynamics and virulence of plant pathogenic microorganisms in a favourable way.

\section{Wicked behaviour}

Soil-borne plant pathogens are the reason for major yield reductions in the production of food, feed, fiber and fuel crops. Two main groups of soil-borne plant pathogens are the nematodes and the fungi (includes the true fungi and the fungal-like oomycetes). In extreme temperate climates, plant pathogenic fungi, oomycetes and nematodes are agronomically more vital than plant pathogenic bacteria, although some bacterial genera (i.e. Pectobacterium, Ralstonia) can also cause large economic damage in some crops. For bacterial pathogens such as, Agrobacterium tumefaciens, Ralstonia solanacearum, Dickeya dadanthi and D. solani, and Pectobacterium carotovorum and $\boldsymbol{P}$. atrosepticum are among the top 10 most notorious groups that infect plants my means of roots. In addition viruses can invade plants through the roots but need vectors such as nematodes or zoosporic fungi to penetrate the root tissue. Evaluated to our acceptance, the role of rhizodeposits in the dialogues exchange of symbionts and plants, information on root exudates that activate and attract soil-borne plant pathogens is more scant and fragmented. The limited information on the communication between plants and root 
pathogens is largely due to miserable understanding of the complicated physical-chemical conditions in soil and rhizosphere environments. Hence, upgraded analytical skills to interpret the chemistry of rhizodeposits and their spatiotemporal production and division patterns, communally termed 'ecometabolomics' will be helpful to resolve the dialogues between pathogens and plant roots (Berendsen et al. 2012, Mendes et al. 2016).

The disease manifestations linked to human pathogen contamination of fresh plant produce are an emerging concern worldwide. On the other hand, salmonellosis is hugely linked to contaminated horticultural products, including fruits, lettuce, cabbage and other raw salad vegetables. Similarly, with increased frequency Escherichia coli O157:H7, the causal agent of the life-threatening hemorrhagic colitis and hemolytic uremic syndrome, has been isolated from fresh food products. A series of studies have clearly shown that the entry of human pathogenic bacteria to the food production chain can have nasty effect not only after harvest but also during the pre-harvest (occur via contaminated manure and irrigation water, animals, or seeds). Moreover, their ability to exist in soils and to colonize the plant explains that human pathogenic bacteria are not wholly adapted to proliferate and live in the animal gastrointestinal tracts.

Few opportunistic human pathogens know how to tactfully behave in the rhizosphere. We know about the 'authentic' human pathogens like Salmonella enteric, Serovar typhimurium and $\boldsymbol{E}$. coli $\mathbf{0 1 5 7 : H 7 , ~ n e x t ~ t o ~ t h e s e ~ t h e ~ p l a n t ~ e n v i r o n m e n t ~ i s ~ a ~ s l o t ~ f o r ~ p a t h o g e n s ~ t h a t ~ c a u s e ~}$ diseases only in devitalized or immune-compromised humans. These facultative human pathogens act as servitor with significant case:fatality ratios in patients in Europe and Northern America and their impact on human health has increased consequently. Various wild and cultivated plant species have shown to host opportunistic human pathogens in the rhizosphere, in particular Burkholderia cepacia, Pseudomonas aeruginosa and Stenotrophomonas maltophilia. However, Bacillus cereus, Proteus vulgaris, bacterial species that cause skin, 
wound and urinary tract infections can be casted in rhizosphere environments. Even though many studies have highlighted the existence of opportunistic human pathogens in the rhizosphere, and their relative virulence to clinical counterparts is not much known. With this context, in an experiment by Kumar et al., on $\boldsymbol{P}$. aeruginosa PaBP35, a strain isolated from the shoots of black pepper plants (grown in a remote rain forest in Southern India), identified relatedness that originated from clinical habitats. A panel of discriminatory genotyping methods and polyphasic analyses revealed that strain PaBP35 was resistant to multiple antibiotics, cultivated at temperatures up to $41^{\circ} \mathrm{C}$, produced rhamnolipids, hydrogen cyanide and phenazine antibiotics, expressed cytotoxicity on mammalian cells and caused infection in an acute murine airway infection model (Kumar et al., 2013). Before this was elicited, plantassociated $P$. aeruginosa M18 was more prone to several antimicrobial agents and easily removed in a mouse acute lung infection model than the clinical strain P. aeruginosa LESB58. Both the above experiments highlighted the versatile feature and adaptive behaviour of $P$. aeruginosa and exemplified that the strains from unlike environments can differ in accompanying genome regions, genome expression profiles, virulence activities and antibiotic resistance band. The occurrence of human pathogenic bacteria in the rhizosphere assigned to several factors, such as the high nutritional content, protection from UV radiation, and the accessibility of dispersal water films for obstructing desiccation. Others have argued that plentiful and highly diverse native rhizosphere microflora stand like a strong barrier against the invasion of human pathogens. The growth of $\boldsymbol{S}$. enterica and $\boldsymbol{E}$. coli $\mathbf{0 1 5 7 : H 7}$ on roots of Arabidopsis thaliana is strongly repressed by a plant-associated strain of Enterobacter asburiae. Nevertheless, a lot of human pathogenic bacteria can be highly competitive for uptake of nutrients and produce various antimicrobial metabolites to colonize and propagate on plant surfaces when the indigenous microflora is present. For example, P. aeruginosa was observed to be a superb colonizer of the wheat rhizosphere. Also clinical and plant-associated 
Stenotrophomonas strains stimulated root growth and root hair development and even very well colonized the strawberry rhizosphere. B. cepacia strains caused disease in a lung infection model was also virulently active on alfalfa. The mechanisms engaged in rhizosphere colonization and antimicrobial activity of human pathogenic bacteria seems to be similar to the mechanisms of virulence and colonization of human tissues. Several pathogenic genes as well as genes related to carbon utilization were regulated in presence of lettuce root exudates in $S$. enteric, and the root exudates trigger chemotaxis switching on genes that play a role in adherence. Among several other genes and including all traits identified in association of human pathogens to plant surface, engage the production of fimbriae, adhesins, and capsule (Berendsen et al. 2012, Mendes et al. 2016, Turner et al. 2013).

\section{Prospects and Outlook}

The overwhelming approach for knowing the exchange of dialogues has expanded within rhizomicrobiome they have an impact on plant as well as human health and is able to influence the structure of the microbial society. The signaling shared by plant root- soil interface and host human with microorganisms dwelling is although lagging behind with many unopened facts, is likely be the subject of many studies in the near future.

Our understanding will become clearer through few more suggested methodologies such as, detecting and quantifying the plant and microbial exudates as well as their effects on gene transcription and translation. Furthermore, cutting-edge techniques like metagenomics metatranscriptomics and metaproteomics which can provide insights into the specific expression and translation of genetic loci in the rhizomicrbiome, besides different imaging mass spectrometry approaches will further allow direct visualization and quantification of specific chemical transfers involving interactions.

The researches on rhizomicrobiome signalling will disclose new avenues to increase crop productivity and reduce the use of agrochemicals. An example could be via the modification 
of plant metabolic pathways of signals as a mode to change the rhizobiome inhabitants. Selection and extraction of rhizosphere signaling molecules could also be used directly as chemical cues will foster as beneficial rhizomicrobiome. These ways can be devised for a more sustainable agriculture to enrich the rhizomicrobiome for the beneficial members, thereby escalating plant resistance to varying stresses.

Another challenging space is how to check human pathogen proliferation in plant environments to critical doses causing human disease. Therefore, a improved understanding of the factors and hints that enable human pathogens to find a suitable niche on plant surfaces is needed to safeguard human health. To maintain the limitations of plant and human pathogens, varying and balanced trickeries should be developed that transmit the rhizobiome signals in favour of microorganisms that avoid pathogens to germinate, grow, attach, and invade the root tissue. One potential approach is to initiate plant breeding programs untying the molecular level of interactions between plant lines and beneficial members of the rhizomicrobiome. The rhizosphere is measured to be familiar field for ecologists, molecular biologists and plant biologists to further study these new outlooks of interactions stirring in this complex zone. Keeping in mind few proposed approaches and studies we can explore profusely in the wide scope of signalling via rhizobiome.

\section{References}

Ahmed, M.A. Kroener, E. Benard, P. Zarebanadkouki, M. Kaestner, A. Carminati, A. 2016. Drying of mucilage causes water repellency in the rhizosphere of maize: measurements and modelling. Plant Soil 407:161-171.

Bakker, P.A. Berendsen, R.L. Doornbos, R. F. Wintermans, P. C. A. Pieterse, C.M. 2013. The rhizosphere revisited:root microbiomics. Frontiers in Plant science 165(4).

Berendsen, R.L. Pieterse, C.M. Peter, Bakker, P.A. 2012. The rhizosphere microbiome and plant health. Trends in Plant Science 17(8).

Halder, S. Senguta, S. 2015. Plant-microbe Cross-talk in the Rhizosphere: Insight and Biotechnological Potential. The Open Microbiology Journal 9:1-7. 
Inceoglu, O. VanOverbeek, L.S. Salles, J.F. VanElsas, J.D. 2013. The normal operating range of bacterial communities in soil used for potato cropping. Appl.Environ.Microbiol 79:1160-1170.

Jha, B. Gontia, I. Hartmann, A. 2012. The roots of the halophyte Salicornia brachiata are a source of new halotolerant diazotrophic bacteria with plant growth-promoting potential. Plant Soil 356: 265-277.

Jones, D.L. Nguyen, C. Finlay, R.D. 2009. Carbon flow in the rhizosphere: carbon trading at the soil-root interface. Plant Soil 321: 5-33.

Kumar, A. Munder, A. Aravind, R. Eapen, S.J. Tummler, B. Raaijmakers, J.M. 2013. Friend or foe: genetic and functional characterization of plant endophytic Pseudomonas aeruginosa. Environ Microbiol 15: 764-779.

Li, B. Li, Y. Wu, H. M. Zhang, F. Li, C.J. Li, X. Lambers, H. Li, L. 2016. Root exudates drive interspecific facilitation by enhancing nodulation and N-2 fixation. Proc Natl Acad Sci U S A 113:6496-6501.

Lugtenberg, B. Kamilova, F. 2009. Plant-growth-promoting rhizobacteria. Annu Rev Microbiol 63: 541-556.

Mendes, R. Garbeva, P. Raaijmakers, Jos M. 2013. The rhizosphere microbiome: significance of plant beneficial, plant pathogenic, and human pathogenic microorganisms. FEMS Microbiol Rev 37:634-663.

Mommer, L. Hinsinger, P. Combaret, C.P. Visser, E.J.W. 2016. Advances in the rhizosphere: stretching the interface of life. Plant Soil 407:1-8.

Peterson, Andrew H. 2013. Advances in Botanical Research: Genomes of Herbaceous Land Plants. Volume 69. Chapter 11. $1^{\text {st }}$ ed. Elsevier Publishing.

Quiza, L. St-Arnaud, M. Yergeau, E. 2015. Harnessing phytomicrobiome signaling for rhizosphere microbiome engineering. Front. Plant Sci. 6:507.

Robroek, B.J.M. Albrecht, R.J.H. Hamard, S. Pulgarin, A. Bragazza, L. Buttler, A. Jassey, V.E. 2016. Peatland vascular plant functional types affect dissolved organic matter chemistry. Plant Soil 407:135-143.

Siddikee, M. Chauhan, P. Anandham, R. Han, G.H. 2010. Isolation, characterization, and use for plant growth promotion under salt stress, of ACC deaminase-producing halotolerant bacteria derived from coastal soil. J Microbiol Biotechnol 20: 1577-1584. 
Tanaka, N. Nishida, S. Kamiya, T. Fujiwara, T. 2016. Large-scale profiling of brown rice ionome an ethyl methanesulphonate-mutagenized hitomebore population and identification of high- and low-cadmium lines. Plant Soil 407:109-117.

Turner, T.R. James, E.K. Poole, P.S. 2013. The plant microbiome. Genome Biology 14:209.

van Dam, N.M. Bouwmeester, H.J. 2016. Metabolomics in the rhizosphere: tapping into belowground chemical communication. Trends Plant Sci 21:256-265.

Venturi, V. Keel, C. 2016. Signalling in the rhizosphere. Trends Plant Sci 21:187-198.

Vetterlein, D. Doussan, C. 2016. Root age distribution: how does it matter in plant processes? a focus on water uptake. Plant Soil 407:145-160.

Zhang, D. Zhang, C. Tang, X. Li, H. Zhang, F. Rengel, Z. Whalley, W.R. Davies, W.J. Shen, J. 2016. Increased soil phosphorus availability induced by faba bean root exudation stimulates root growth and phosphorus uptake in neighbouring maize. New Phytol 209:823831. 